\title{
Holographic Calculation of Boundary Entropy
}

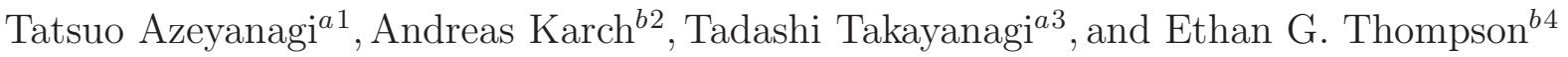 \\ ${ }^{a}$ Department of Physics, Kyoto University, Kyoto 606-8502, Japan \\ ${ }^{b}$ Department of Physics, University of Washington, Seattle, Wa, 98195, USA
}

\begin{abstract}
We use the holographic proposal for calculating entanglement entropies to determine the boundary entropy of defects in strongly coupled two-dimensional conformal field theories. We study several examples including the Janus solution and show that the boundary entropy extracted from the entanglement entropy as well as its more conventional definition via the free energy agree with each other. Maybe somewhat surprisingly we find that, unlike in the case of a conformal field theory with boundary, the entanglement entropy for a generic region in a theory with defect carries detailed information about the microscopic details of the theory. We also argue that the g-theorem for the boundary entropy is closely related to the strong subadditivity of the entanglement entropy.
\end{abstract}

\footnotetext{
${ }^{1}$ e-mail:aze@gauge.scphys.kyoto-u.ac.jp

${ }^{2}$ e-mail:karch@phys.washington.edu

${ }^{3}$ e-mail:takayana@gauge.scphys.kyoto-u.ac.jp

${ }^{4}$ e-mail:egthomps@u.washington.edu
} 


\section{Introduction}

Quantum systems with defects or boundaries often show interesting physical behaviors. For example, impurities in various materials have been one of the major subjects of research in condensed matter physics. In such systems, we expect that the whole system (both the bulk and the boundary) flows towards a fixed point of the renormalization group in the low energy limit, where we can employ the powerful method of conformal field theory. The boundary or defect preserves a part of the conformal symmetry at the fixed point.

One of the important quantities which characterizes properties of the defects or boundaries is known as the boundary entropy (or, equivalently, the ground state degeneracy $g$ ) [1. This measures the degrees of freedom localized at a given defect and is a boundary analogue of the central charge $c$. Recently, it has also been pointed out that the boundary entropy can be regarded as the finite part of the entanglement entropy [2].

In general, the theory is strongly interacting at the RG fixed point and sometimes it is very difficult to calculate physical quantities like the boundary entropy. However, if the theory has a holographic dual, we can compute many quantities rather simply by using the dual gravity description. The most tractable examples will be the ones for which we can apply the AdS/CFT correspondence.

The purpose of this paper is to holographically compute the boundary entropy of $2 \mathrm{~d}$ conformal field theories with defects using several methods (for early discussions refer to [3]). The holographic calculation of entanglement entropy has been recently formulated

[4, 5]. This allows us to find the boundary entropy from the entanglement entropy, in addition to using a probe computation of the boundary entropy at high temperature.

The organization of this paper is as follows. In section 2, we present a brief summary of the definition and properties of the boundary entropy. We will also work out a close relation between the g-theorem and the strong subadditivity of entanglement entropy. In section 3, we perform the holographic computations of the boundary entropy using both probe configurations and fully back-reacted geometries (in particular for the Janus solution). In section 4 we summarize our conclusions. In appendix A, we present the calculations of two point functions and the boundary entropy of a free scalar field in the presence of the interface. 


\section{Boundary Entropy and Entanglement Entropy}

In this paper, we are interested in two dimensional conformal field theories (2d CFTs) in the presence of a conformal defect. If we define the time and space coordinate by $(t, x)$, then we can consider a time-like defect which is situated at $x=0$. The defect is called conformal if a linear combination of two Virasoro symmetries in the bulk is preserved. We will refer to a CFT with such a defect as a defect conformal field theory (DCFT) (e.g. see the review part of [6]). Generically, there are extra propagating degrees of freedom localized on the defect. However, it is also possible to construct a system with no new degrees of freedom on the defect. Such a theory is called an interface CFT (ICFT). A simple example of an ICFT is a compactified scalar field $\phi(t, x)$ whose radius jumps at the defect. An interesting quantity which characterizes a system with a conformal boundary or defect is the boundary entropy, $S_{b d y}$. $S_{b d y}$ is related to the ground state degeneracy $g$ [1] as we explain below.

\subsection{Boundary Entropy and the $g$-function}

Consider a $2 \mathrm{~d}$ CFT with periodic Euclidean time, $t \sim t+2 \pi$. We assume $x$ is also compactified on a large circle with radius $L \gg 1$. When we introduce a defect at $x=0$, the partition function of this system on a torus behaves as

$$
\lim _{L \rightarrow \infty} Z_{\text {torus }}=e^{-L E_{0}+S_{\text {bdy }}}
$$

where $E_{0}$ is the ground state energy when we regard $x$ as the Euclidean time direction. The quantity $S_{b d y}$ is called the boundary entropy. This is motivated by the observation that $S_{b d y}$ is the entropy when we artificially treat $L$ as the temperature and $-\frac{\log Z_{\text {torus }}}{L}$ as the free energy. Originally, the boundary entropy was defined in a 2 d system with a conformal boundary in [1]. However, the DCFT can be equivalently described by two copies of the system with boundary via the doubling trick, as discussed, for example, in [6] or in appendix $\mathrm{A}$ of this paper.

The quantity $g$, defined by

$$
g \equiv e^{S_{b d y}}
$$

represents the ground state degeneracy. We can extend the idea of the boundary entropy to non-conformal systems and define the $g$-function. According to the $g$-theorem [1], the $g$-function is a monotonically decreasing function with respect to the length scale $l$,

$$
\frac{d}{d l} \log g(l) \leq 0,
$$

in analogy to the $c$-function and $c$-theorem. 


\subsection{Boundary Entropy from Entanglement Entropy}

Recently, it was found that the boundary entropy is actually related to a physical entropy, the entanglement entropy [2]. To define the entanglement entropy, we first divide the system into two parts $A$ and $B$. Accordingly, the total Hilbert space is factorized as $H=H_{A} \otimes H_{B}$. Next we introduce the reduced density matrix $\rho_{A}=\operatorname{Tr}_{B} \rho$ for the subsystem $A$ by tracing out $H_{B}$. Finally, the entanglement entropy is defined as the von-Neumann entropy for $\rho_{A}$

$$
S_{A}=-\operatorname{Tr} \rho_{A} \log \rho_{A} .
$$

Consider an infinitely long system and define the subsystem $A$ by the finite interval with length $l$. The subsystem $B$ is defined to be the complement of $A$. Then the entanglement entropy $S_{A}$ can be computed to be [7]

$$
S_{A}=\frac{c}{3} \log \frac{l}{a},
$$

where $c$ is the central charge of the total system and $a$ is the UV cut off (i.e. lattice spacing).

In the presence of a conformal boundary with boundary entropy $\log g$, this is modified as follows 2

$$
S_{A}=\frac{c}{6} \log \frac{l}{a}+\log g
$$

Because the boundary cuts off half of the space, we have the coefficient $\frac{c}{6}$ instead of $\frac{c}{3}$.

When we consider a conformal defect which is situated at the middle of the interval $A$, we can regard the system as two copies of a BCFT by the doubling trick. This leads to the following result

$$
S_{A}=\frac{c}{3} \log \frac{l}{a}+\log g .
$$

To see the relation (2.7) quickly, let us remember that in the $2 \mathrm{~d}$ CFT $S_{A}$ can be found from the formula

$$
S_{A}=-\left.\frac{\partial}{\partial n} \operatorname{Tr} \rho_{A}^{n}\right|_{n=1}=-\left.\frac{\partial}{\partial n}\left[\frac{Z_{n}}{\left(Z_{1}\right)^{n}}\right]\right|_{n=1},
$$

where $Z_{n}$ is the partition function on the n-sheeted Riemann surface with a cut along the interval $A$ [2]. The important point is that both the original two dimensional space and the $n$-sheeted one both have a single connected boundary. Thus the ratio $\frac{Z_{n}}{\left(Z_{1}\right)^{n}}$ is proportional to the factor $g^{1-n}$, which leads to the formula (2.7).

On the other hand, if the defect is not located at the midpoint of the interval, the entanglement entropy cannot be determined only from $c$ and $g$, but rather it depends on the details of the theory. This is because we cannot relate this DCFT setup to the BCFT 
setup by the folding trick, as the quantity we are interested in does not have the reflection symmetry about the defect. In other words, it is not possible to find a conformal map from the $n$-sheeted Riemann surface defined by $v^{n}=\frac{w-l_{1}}{w+l_{2}}$ with the defect at Re $w=0$, to a single complex plane $\mathbf{C}$ with a straight defect line except for $l_{1}=l_{2}$, which means that the defect is at the midpoint of the interval.

\subsection{Strong Subadditivity and g-theorem}

It is intriguing to see if we can obtain useful properties of the boundary entropy from the basic properties of entanglement entropy. One of the most important inequalities satisfied by any entanglement entropy is the strong subadditivity constraint (e.g. refer to the review part of [8, 9]). It is represented by the inequality

$$
S_{A}+S_{B} \geq S_{A \cap B}+S_{A \cup B}
$$

The holographic derivation of strong subadditivity has been given in [10, 9].

It was shown in [8] that the entropic analogue of the $c$-theorem follows from this relation. Therefore, it is natural to ask if the $g$-theorem can also be derived from this condition. Let us start with a simple setup (see fig:1) in a defect CFT. $A$ is defined by $\left[-l_{a}-l_{c}, l_{a}+l_{c}\right]$ and $B$ is defined by the two intervals $\left[-l_{a}-l_{b}-l_{c},-l_{a}\right]$ and $\left[l_{a}, l_{a}+l_{b}+l_{c}\right]$. In this case, by substituting (2.7) into the strong subadditivity constraint (2.9), we obtain

$$
\begin{aligned}
& \frac{c}{3} \log 2\left(l_{a}+l_{c}\right)+\log g\left(2\left(l_{a}+l_{c}\right)\right)+2 \cdot \frac{c}{3} \log \left(l_{b}+l_{c}\right) \\
& \quad \geq 2 \cdot \frac{c}{3} \log l_{c}+\frac{c}{3} \log \left(2\left(l_{a}+l_{b}+l_{c}\right)\right)+\log g\left(2\left(l_{a}+l_{b}+l_{c}\right)\right) .
\end{aligned}
$$

Then in the limit $l_{b} \rightarrow 0$ we find

$$
\left.\frac{d}{d l} \log g(l)\right|_{l=2\left(l_{a}+l_{c}\right)} \leq \frac{c}{6}\left(\frac{2}{l_{c}}-\frac{1}{l_{a}+l_{c}}\right) .
$$

By taking the limit $l_{a} \rightarrow 0$, we obtain the bound

$$
\frac{d \log g(l)}{d l} \leq \frac{c}{3 l}
$$

Even though this is not enough to prove the $g$-theorem (2.3), we can at least say that the $g$-theorem is non-trivially consistent with the strong subadditivity.

To see the relation to the g-theorem more clearly, we need to cancel the log terms in (2.10). This can be done by considering a relativistic setup as in fig.2. Notice that 
by requiring Lorentz invariance, the Hilbert space $H_{A}$ for $A$ depends only on the causal future (or past) of $A$ and remains the same under any deformation which preserves it. If the Lorentz invariant length of $A$ is denoted by $|A|$, we can easily show $|A| \cdot|B|=$ $|A \cap B| \cdot|A \cup B|$. Owing to this relation, the authors in [8] were able to prove the $c$-theorem from the strong subadditivity condition for this setup. Indeed, strong subadditivity leads to

$$
S\left(l_{1}\right)+S\left(l_{2}\right) \geq S\left(l_{3}\right)+S\left(l_{4}\right),
$$

where we assume $l_{1} l_{2}=l_{3} l_{4}$ and $l_{4} \leq l_{1}, l_{2} \leq l_{3}$. This is equivalent to the concavity of the entropy as a function of $\log l$ i.e.

$$
l \frac{d}{d l}\left(l \frac{d S(l)}{d l}\right) \leq 0 .
$$

Noting that $c(l)=3 l \frac{d S(l)}{d l}$, it is clear from (2.5) that the inequality (2.14) is precisely the entropic $c$-theorem.

Now we return to the relation to the $g$-theorem and thus we assume that the bulk region is conformal. If we again employ the choice of subsystems and the defect line as described in fig, 2, the bulk log terms are completely canceled out. Since the other part of the entanglement entropy can be regarded as an entropic g-function, we simply obtain

$$
\log g(A) \geq \log g(A \cup B)
$$

This indeed agrees with the g-theorem in a particular case. In this way, we have learned that strong subadditivity for the entanglement entropy is closely related to the $g$-theorem for DCFTs. We leave a further study of this issue as a future problem.

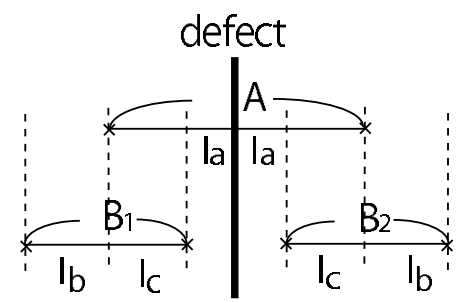

Figure 1: The simple setup of $A$ and $B$ at a common fixed time. Notice that both $A$ and $B$ live in the same one dimensional space. 


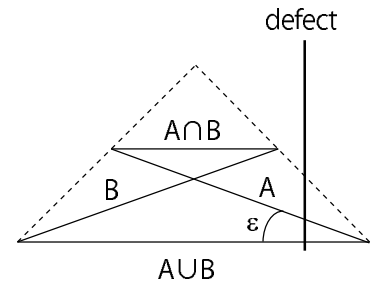

Figure 2: The relativistic setup of $A$ and $B$. The vertical and horizontal directions represent the time and space coordinates, respectively. The dotted light-like triangle delimits the causal future.

\section{Holographic Boundary Entropy of Defect}

An interesting class of $2 \mathrm{~d}$ CFTs, in which the boundary entropy can be calculated using its relation to the entanglement entropy, is those $2 \mathrm{~d}$ CFTs which have a dual description in terms of a higher dimensional gravitational theory on an asymptotically $\mathrm{AdS}_{3}$ background. A method to calculate entanglement entropies in theories with gravitational duals has been found in [4, 5]. This equates the entanglement entropy of a spatial region $\mathcal{M}$ in the field theory bounded by $\partial \mathcal{M}$ with $\frac{A}{4 G_{N}^{(3)}}$, where $A$ is the area of a minimal area surface ending on $\partial \mathcal{M}$ and in a constant time slice of the $3 \mathrm{~d}$ bulk.$G_{N}^{(3)}$ is the $3 \mathrm{~d}$ Newton constant. We will apply this formula here to known examples of DCFTs with a gravitational dual.

In particular, we will look at three different systems. The first system we study is a Randall-Sundrum (RS) like toy model [11, 12] of a brane coupled to gravity, which for a certain range of tensions has a dual description in terms of a DCFT. Since this model, in its simplest form, has not been embedded in string theory or any other consitent theory of quantum gravity, we don't know precisely what the DCFT is (and whether it exists at all). But the advantage is that in this case we can calculate both the entanglement entropy as well as (in the "probe" limit of small tension) the high temperature free energy, confirming that the two alternative definitions of the boundary entropy do indeed agree.

The second model we look at is the Janus solution [13, 14]. In this example, one once more knows the full bulk geometry and can calculate the entanglement entropy. For Janus the dual DCFT is known and is of the interface type. We can calculate the boundary entropy also in the limit of weak coupling, where the calculation is tractable on the field theory side. To leading order in the parameter controlling the jump across the interface, we observe agreement between weak and strong coupling.

Last, but not least, we look at defects with localized matter. These systems often 
have a dual description in terms of a probe brane embedded in the $\mathrm{AdS}_{3}$ space. In these scenarios we don't have access to the entanglement entropy without controlling the backreaction. However, we can calculate the boundary entropy via the high temperature free energy, as was already pointed out in [3]. The dual field theory is once more well understood and we can compare weak and strong coupling answers.

\subsection{Defect in a Toy Model}

Our first example of a 3-dimensional geometry with a dual description in terms of a DCFT arises as a solution to the RS [11] action of a $2 \mathrm{~d}$ brane with tension $\lambda$

$$
S=\frac{1}{16 \pi G_{N}^{(3)}} \int d^{3} x \sqrt{-g}\left(R+\frac{2}{R_{A d S}^{2}}\right)-\lambda \int d^{2} x \sqrt{-g_{I}},
$$

where $g_{I}$ is the determinant of the induced metric on the $2 \mathrm{~d}$ slice spanned by the brane. Without the brane the solution to this system would be $\mathrm{AdS}_{3}$ with curvature radius $R_{\operatorname{AdS}}$. For branes with a tension $\lambda$ less than a critical value $\lambda_{*}=\frac{1}{4 \pi G_{N}^{(3)} R_{A d S}}$ one can find solutions which have a brane with an $\mathrm{AdS}_{2}$ geometry and hence precisely preserve the symmetries expected from a dual DCFT. No embedding of the system in this simple form into string theory is known. Assuming that it makes sense as a quantum theory, the observables in this theory have the interpretation of correlation functions in some DCFT [12]. For this toy model we do not have an alternative definition of the DCFT.

\subsubsection{The Background Solution}

To construct the solution, consider the $d+1$ dimensional asymptotic AdS background

$$
d s^{2}=R_{A d S}^{2}\left(d y^{2}+e^{2 A(y)}\left(d s_{A d S_{d}}\right)^{2}\right) .
$$

The pure AdS corresponds to $e^{A(y)}=\cosh y$.

We are interested in the dual of a $2 \mathrm{~d}$ CFT so we set $d=2$. Then we can write $\left(d s_{A d S_{d=2}}\right)^{2}=-\cosh ^{2} r d t^{2}+d r^{2}$. In the ordinary global coordinate we can rewrite as follows

$$
d s^{2}=R_{A d S}^{2}\left(-\cosh ^{2} \rho d t^{2}+d \rho^{2}+\sinh ^{2} \rho d \theta^{2}\right) .
$$

For pure $\mathrm{AdS}_{3}$, the coordinates are related to each other via

$$
\cosh y \cosh r=\cosh \rho, \quad \sinh y=\sinh \rho \sin \theta .
$$

Using these global coordinates the geometry on which the dual CFT lives is actually a circle and not just a line. There are two defects at $\theta=0$ and $\theta=\pi$, corresponding to 
the boundary points at fixed $y$ but infinite $r$. In the presence of a codimension one defect with tension $\lambda$, the equation of motion becomes

$$
-1+\left(A^{\prime}\right)^{2}+A^{\prime \prime}=8 \pi G_{N}^{(3)} R_{A d S} \lambda \delta(y),
$$

where we assumed that the brane is situated at $y=0$. This can be solved by

$$
e^{A(y)}=\cosh \left(|y|-y_{*}\right)
$$

The constant $y_{*}$ is defined by

$$
\tanh y_{*}=4 \pi G_{N}^{(3)} R_{A d S} \lambda \text {. }
$$

The spacetime with the backreaction due to the brane becomes two copies of the partial AdS spacetime defined by $-y_{*}<y<\infty$ in the AdS sliced coordinates (3.2).

\subsubsection{Boundary Entropy from Entanglement Entropy}

As mentioned in the beginning of this section, the holographic recipe for calculating entanglement entropies is to find at a fixed time $t$ the minimal area surface in the bulk that ends on the boundary of the region whose entropy we want to calculate. In the case of a 3-dimensional bulk spacetime this minimal spatial area is simply a geodesic. If we concentrate on the largest region in the field theory that is symmetric around the defect we are looking for a geodesic that connects the boundary points $\theta=-\pi / 2$ and $\theta=\pi / 2$. That is, in the coordinate system of eq. (3.2) we want to connect the point $r=0$ at $y=+\infty$ with the point $r=0$ at $y=-\infty$. By symmetry it is easy to see that the geodesic is $r=0$. We will return to this in more detail later when we look at asymmetric regions.

For this longest geodesic we can easily calculate the extra length $\Delta L$ induced by the defect brane as follows

$$
\Delta L=2 R_{A d S} y_{*} .
$$

Thus the extra contribution to the entanglement entropy becomes

$$
\Delta S_{A}=\frac{R_{A d S} y_{*}}{2 G_{N}^{(3)}} .
$$

In the probe limit, the brane tension is very small $\left(y_{*} \ll 1\right)$ and we can approximately obtain

$$
\Delta S_{A}=2 \pi R_{A d S}^{2} \lambda
$$

$\log g=\Delta S_{A}$ can directly be identified as the boundary entropy of the dual DCFT. 


\subsubsection{Boundary Entropy from Free Energy}

Without the brane, turning on a finite temperature corresponds to replacing the $\mathrm{AdS}_{3}$ solution in the bulk with a BTZ black hole,

$$
d s^{2}=-h\left(r_{B T Z}\right) d t^{2}+\frac{d r_{B T Z}^{2}}{h\left(r_{B T Z}\right)}+r_{B T Z}^{2} d \theta^{2}
$$

with $h\left(r_{B T Z}\right)=r_{B T Z}^{2}-\mu+1$. For simplicity we switched to units in which the curvature radius $R_{A d S}=1$. For $\mu=0$ this is simply global AdS and reduces to eq. (3.3) by a change of coordinates $\sinh (\rho)=r_{B T Z}$. The BTZ black hole has a horizon at $r_{H}$ such that $r_{H}^{2}=\mu-1$. The temperature of the black hole is given by $T=\frac{h^{\prime}\left(r_{H}\right)}{4 \pi}=\frac{r_{H}}{2 \pi}$.

In order to study the free energy of the DCFT at finite temperature we need to find the generalization of the BTZ black hole with the backreaction of the brane included. This is a difficult problem and no solutions are known. However, in the small tension limit the change of the geometry due to the brane can be neglected. As a power series expansion in the tension of the brane, the leading contribution comes from the on-shell action of the brane probe which minimizes its worldvolume in the fixed BTZ black hole background geometry. This is in complete analogy to the calculation that allows one to calculate order $N_{f} N_{c}$ corrections to the order $N_{c}^{2}$ free energies in a theory with a large number of colors $N_{c}$ and a finite number of flavors $N_{f}$ using probe branes [15]. This technique has been first used for a free energy calculation in [16] and has been confirmed by many calculations since.

The action describing the embedding of the brane is proportional to the worldvolume of the brane,

$$
S_{\text {probe }}=-\lambda \int d^{2} x \sqrt{-g_{I}}
$$

A simple embedding is given by the union of $\theta=0$ and $\theta=\pi$. This is the finite temperature generalization of the probe stretching straight across the $\mathrm{AdS}_{3}$ space along the central $y=0$ slice in the coordinate system of eq. (3.2). It is the minimal action configuration which satisfies the boundary conditions that the probe ends on the defect:5, which are located at $\theta=0$ and $\theta=\pi$. The induced metric on this brane is $d s^{2}=$ $-h d t^{2}+\frac{d r_{B T Z}^{2}}{h}$ and so the determinant of the induced metric is 1 . Wick rotating to Euclidean signature and regulating the on-shell action by simply subtracting the zero

\footnotetext{
${ }^{5}$ Alternatively we can work in the analog of Poincare Patch coordinates where we drop the 1 from $h(r)$ and think of $\theta$ as living on the real line as opposed to on a circle. In this case we describe a single defect at $\theta=0$.
} 
temperature answer we get for the free energy associated with a single defect

$$
F=-T S_{\text {on-shell }}=\lambda \lim _{r_{c} \rightarrow \infty}\left(\int_{r_{H}}^{r_{c}} d r-\int_{0}^{r_{c}} d r\right)=-\lambda r_{H}=-2 \pi T \lambda .
$$

The entropy now can be calculated using the standard relation

$$
S=-\frac{\partial F}{\partial T}=2 \pi \lambda
$$

Restoring the curvature radius $R_{A d S}$, this is in perfect agreement with the answer for the boundary entropy we got from the entanglement entropy, eq. (3.10).

\subsection{Boundary Entropy and Janus Solution}

It is well-known that the near horizon limit of the D1-D5 system is type IIB string theory on $\mathrm{AdS}_{3} \times S^{3} \times T^{4}$. We assume that there are $Q_{1}$ D1-branes and $Q_{5}$ D5-branes in this system. The $\mathrm{AdS}_{3} / \mathrm{CFT}_{2}$ correspondence claims that the string theory in this background is dual to the $(4,4)$ superconformal sigma model whose target space is the symmetric product $\left(T^{4}\right)^{Q_{1} Q_{5}} / S_{Q_{1} Q_{5}}$. We would like to deform this CFT so that it includes a conformal defect. In particular, we are interested in an interface which separates two regions with $T^{4}$ of different radii. We assume that the radius of $T^{4}$ changes from $R_{+}$to $R_{-}$.

Recently, a 3 dimensional gravity background has been constructed [14] that is a particular example of the Janus solutions. The supergravity metric in the Einstein frame is

$$
d s_{I I B}^{2}=e^{\frac{\phi}{2}}\left(d s_{(3)}^{2}+d \Omega_{3}^{2}\right)+e^{-\frac{\phi}{2}} d s_{T^{4}}^{2} .
$$

The $(2+1)$ dimensional part $d s_{(3)}^{2}$ is described by the Einstein-Hilbert action plus a scalar field $\phi$ (i.e. in Einstein frame). This is because $\sqrt{-g^{(10)}} R^{(10)}=\sqrt{-g^{(3)}} R^{(3)}$. For the Janus solution, the 3D metric is explicitly given by

$$
d s_{(3)}^{2}=R_{A d S}^{2}\left(d y^{2}+f(y) d s_{A d S_{2}}^{2}\right),
$$

where the function $f(y)$ is found to be

$$
f(y)=\frac{1}{2}\left(1+\sqrt{1-2 \gamma^{2}} \cosh 2 y\right) .
$$

Also, the two asymptotic values of the dilaton $\phi_{ \pm} \equiv \phi( \pm \infty)$ are found to be

$$
\phi_{ \pm}=\phi_{0} \pm \frac{1}{2 \sqrt{2}} \log \left(\frac{1+\sqrt{2} \gamma}{1-\sqrt{2} \gamma}\right) .
$$


For $\gamma=0$ the dilaton is constant, $\phi=\phi_{0}$, and the metric reduces to pure AdS in the coordinate system of eq. (3.2).

The geodesic distance $L$ is needed in order to compute the holographic entanglement entropy. To obtain it, we have to be careful about the regularization of the UV divergence. This can be done by expressing the asymptotically AdS metric always in the form $d s_{(3)}^{2} \simeq$ $R_{A d S}^{2} \frac{d z^{2}+d x^{2}-d t^{2}}{z^{2}}$. Then the UV cutoff is always given by $z=\epsilon$. In our case of (3.16) we obtain

$$
\epsilon=e^{-y_{\infty}} \frac{2}{\left(1-2 \gamma^{2}\right)^{\frac{1}{4}}}
$$

In this way, we can find the additional contribution to the entanglement entropy when we put a non-zero value of the Janus deformation $\gamma$ to be

$$
\Delta L=L-L_{\infty}=2 R_{A d S}\left(y_{\infty}(\gamma)-y_{\infty}(0)\right)=R_{A d S} \log \frac{1}{\sqrt{1-2 \gamma^{2}}}
$$

The radius and the 3D Newton constant expressed in terms of the dual $2 \mathrm{~d}$ CFT quantities are given by

$$
R_{A d S}=\sqrt{g_{6}}\left(Q_{1} Q_{5}\right)^{1 / 4} l_{s}, \quad 4 G_{N}^{(3)}=\sqrt{g_{6}}\left(Q_{1} Q_{5}\right)^{-3 / 4} l_{s}, \quad g_{6}=g_{s} \sqrt{\frac{Q_{5}}{Q_{1}}} .
$$

Thus, in the end we obtain the shift of the entanglement entropy as follows:

$$
\Delta S_{A}=\frac{\Delta L}{4 G_{N}^{(3)}}=\frac{Q_{1} Q_{5}}{2} \log \frac{1}{1-2 \gamma^{2}}=Q_{1} Q_{5}\left(\gamma^{2}+\gamma^{4}+\cdots\right) .
$$

We can claim that this finite part which appears in the Janus background actually corresponds to the boundary entropy (or the logarithm of the $g$-function) by applying the relation (2.7).

Now we want to perform the direct computation of the boundary entropy from the CFT side in order to compare with the above result. To treat the defect CFT we need the doubling trick discussed in [6]. Consider again a single compactified scalar $\phi$ in the presence of the interface where the radius of the scalar jumps from $R_{+}$to $R_{-}$. This theory is equivalent to a BCFT with two scalar fields whose radii are $R_{+}$and $R_{-}$. The boundary condition is the Neumann-Dirichlet type (i.e. there is a 'D1-brane' which wraps the diagonal $S^{1}$ in $T^{2}$ ) as we will review in appendix A. Since the $g$-function is proportional to the tension of the D-brane and is T-dual invariant, we obtain (see [17]) the following results for a single boson compactified with the radius $\tilde{R}$

$$
g_{N}=\sqrt{\frac{\tilde{R}}{\sqrt{2 \alpha^{\prime}}}}, \quad g_{D}=\sqrt{\frac{\sqrt{\alpha^{\prime}}}{\sqrt{2} \tilde{R}}}
$$


for Neumann and Dirichlet boundary conditions, respectively. For the Dp-brane wrapped on $T^{p}$ with the B-field (i.e. the gauge flux) we obtain

$$
g=2^{-p / 4} \cdot \operatorname{det}\left(G-B G^{-1} B\right),
$$

where we assume that all torus coordinates have the periodicity $x_{i} \sim x_{i}+2 \pi \sqrt{\alpha^{\prime}}$.

Our system is described by a D1-brane stretching in the diagonal direction of $T^{2}$. This is T-dual to a D2-brane with a gauge flux $B_{12}=1$, which corresponds to a single D0 charge. Plugging in $g_{11}=\frac{1}{R_{1}^{2}}$ and $g_{22}=R_{2}^{2}$, the formula (3.24) leads to

$$
g=\frac{1}{\sqrt{2}} \sqrt{\frac{R_{+}}{R_{-}}+\frac{R_{-}}{R_{+}}}
$$

Indeed we can confirm $g=1$ at $R_{+}=R_{-}$, which corresponds to the absence of the defect. Thus we get 6

$$
\Delta S_{b d y}=\log g=\log \frac{\sqrt{\frac{R_{+}}{R_{-}}+\frac{R_{-}}{R_{+}}}}{\sqrt{2}} .
$$

Then we need to estimate the value of $\frac{R_{+}}{R_{-}}$dual to the Janus solution. First, we notice that the warp factor of the $T^{4}$ part becomes the constant 1 in the string frame because $G_{\mu \nu}^{\text {Einstein }}=e^{-\frac{1}{2} \phi} G_{\mu \nu}^{\text {string }}$. Thus the kinetic term of the $\left(T^{4}\right)^{Q_{1} Q_{5}} / S_{Q_{1} Q_{5}}$ sigma model should be proportional to $\frac{1}{g_{s}}=e^{-\phi}$. Explicitly, this term goes like $\sim e^{-\phi} \int d z^{2} G_{\mu \nu}^{\text {string }} \partial X^{\mu} \bar{\partial} X^{\nu}$. Thus the radius is proportional to $e^{-\phi / 2}$. The ratio $R_{+} / R_{-}$in the Janus solution becomes

$$
\frac{R_{+}}{R_{-}}=\left(\frac{1+\sqrt{2} \gamma}{1-\sqrt{2} \gamma}\right)^{\frac{1}{2 \sqrt{2}}}
$$

We can estimate the boundary entropy as follows

$$
S_{b d y}=4 Q_{1} Q_{5} \log \frac{1}{\sqrt{2}} \sqrt{\left(\frac{1+\sqrt{2} \gamma}{1-\sqrt{2} \gamma}\right)^{\frac{1}{2 \sqrt{2}}}+\left(\frac{1-\sqrt{2} \gamma}{1+\sqrt{2} \gamma}\right)^{\frac{1}{2 \sqrt{2}}}}=Q_{1} Q_{5}\left(\gamma^{2}+\frac{7}{6} \gamma^{4}+\cdots\right)
$$

${ }^{6}$ The boundary entropy of this DCFT very recently has also been studied in [18]. Interestingly, the authors find that such an interface, where the radius of a compact scalar jumps by a finite amount, can increase the entropy by splitting into 2 defects with smaller jumps. This process repeats and ultimately one should obtain infinitely many defects with infinitessimally small jumps. In [18] this property is identified as an instability in the sense of the renormalization group flow. The CFT has relevant operators that drive the RG flow away from the fixed point with a single defect. This only turns into a dynamical instability if we promote the radius of the scalar into a dynamical field. We thus should not expect to see this as an instability in the spectrum of normalizable fluctuations around the Janus geometry with fixed asymptotic behavior, consistent with the positive energy theorem proven for Janus-type solutions in [19]. 
As expected, the boundary entropy in the free theory can also be calculated via the free energy yielding identical results. We present that calculation in appendix A.

Thus the leading term $\left(\sim \gamma^{2}\right)$ from AdS (3.22) agrees with the one from CFT (3.28). Thinking of the Janus field theory in the framework of conformal perturbation theory, as in [20], this agreement hints at a non-renormalization of some correlation functions of the Lagrangian. The relevant correlation functions are those of the Lagrangian with the twist fields that produce the $n$-sheeted Riemann surface, corresponding to $Z_{n}$ in (2.8); refer to [2, 5] for general discussion. Also, as shown in fig 3, the deviations of (3.22) from (3.28) are very small for any value of $\gamma$. We may notice that the boundary entropy in the free field theory is always larger than that in AdS (i.e. at strong coupling), which is natural.

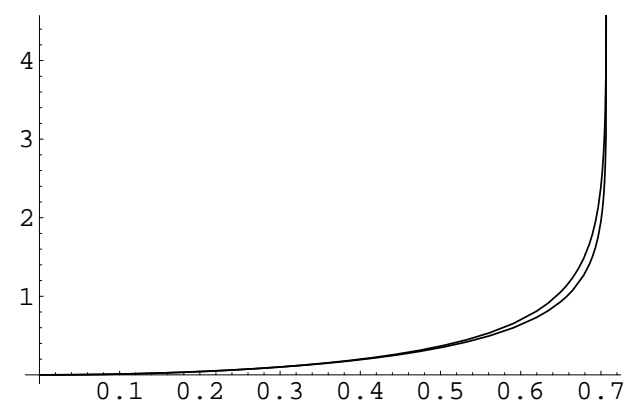

Figure 3: The plot of the boundary entropy from both the AdS and free CFT calculation. We plotted the values of $\frac{S_{b d y}}{Q_{1} Q_{5}}=\frac{\log g}{Q_{1} Q_{5}}$ as a function of $\gamma$. Notice that $\gamma$ can take the values $0 \leq \gamma \leq \frac{1}{\sqrt{2}}$. The upper and lower curve correspond to the free field CFT result (3.28) and AdS result (3.22), respectively. They almost coincide with each other, but there is a small deviation.

\subsection{Probe Computations for D1 D5 System}

Last, but not least, we want to study another set of DCFTs. A whole class of DCFTs can be realized via probe7 D-branes in known AdS backgrounds [21]. For $\mathrm{AdS}_{3}$ such probe branes were first discussed in [22]. In the dual field theory, the probe brane corresponds to adding a finite number of localized matter fields into a CFT with a large number of degrees of freedom, for example $N_{f}$ fundamental hypermultiplets into a large $N_{c}$ gauge theory. Focusing on $2 \mathrm{~d}$ field theories, we can start with the $\mathrm{AdS}_{3} \times S^{3} \times T^{4}$ spacetime

\footnotetext{
${ }^{7}$ Probe brane here refers to the limit where the backreaction of the brane on the geometry is negligible, so that the brane simply minimizes its worldvolume action in a given fixed background. In the field theory this corresponds to the quenched approximation which is justified by a large number of colors limit.
} 
considered in the last subsection and add a probe F1-string on $\mathrm{AdS}_{2}$ or a probe D3 brane on $\mathrm{AdS}_{2} \times S^{2}$ [23, 24]. Without solving for the backreaction of these branes we cannot extract the entanglement entropy. It is however straightforward to obtain the free energy at high temperatures directly from the probe action, just as we did in section 3.1 .3 for the RS brane in the small tension limit.

Consider $M$ F1-strings or $M$ D3 branes in the BTZ black hole background eq. (3.11), since these are the potential supersymmetric probes. These branes have an action which is given by their area just as in the RS toy model. In addition there are also couplings to the background form fields, in particular in the WZ term in the D3 action. We must consider how these terms contribute to the action. In the string theory setup the background is supported by a 3-form RR flux $H$. A convenient gauge choice for the RR 2-form is to take it to be of the form $B_{R R}=B(r) d t \wedge d \theta$. The probe embedding we are looking for is the same $\theta=0$ one we discussed in the RS toy model. Since the pullback of $B_{R R}$ for this embedding is zero it does not contribute to the on-shell action and, as before, we get the free energy (and hence the boundary entropy associated with the defect) simply from the volume of the brane. The result, as in eq. (3.14), is that for a brane of tension $\lambda$ the boundary entropy of the defect dual to the probe brane is

$$
S=2 \pi R_{A d S}^{2} \lambda
$$

For the F1 and D3 branes all we need is to plug in the relevant values of the tension $\lambda_{F 1,3}$ using eq. (3.21). The general formula is that the tension of a Dp brane is $\lambda_{p}=\frac{1}{(2 \pi)^{p}} \frac{1}{g s} \frac{1}{l_{s}^{p+1}}$ and $\lambda_{F 1}=\frac{1}{2 \pi l_{s}^{2}}$ for the fundamental string. With this we get for a single probe brane

$$
\begin{array}{r}
\lambda_{F 1}=\frac{1}{2 \pi l_{s}^{2}}=\frac{g_{s} Q_{5}}{2 \pi R_{A d S}^{2}} \quad \Rightarrow \quad S_{F 1}=g_{s} Q_{5}, \\
R_{A d S}^{2} \lambda_{3}=\frac{R_{A d S}^{2}}{(2 \pi)^{3} g_{s} l_{s}^{4}}=\frac{g_{s} Q_{5}^{2}}{(2 \pi)^{3} R_{A d S}^{2}} \quad \Rightarrow \quad S_{3}=\frac{g_{s} Q_{5}^{2}}{(2 \pi)^{2}} .
\end{array}
$$

For $M$ probe branes we get $M$ times these expressions. A boundary entropy scaling as $M Q_{5}$ is expected for the D3 brane from the weak coupling consideration. This gets enhanced by a power of the 't Hooft couplings $g_{s} Q_{5}$. Such a strong coupling enhancement of the free energy has been seen in other probe systems before, such as the D7 probe that adds flavor to $\mathcal{N}=4$ super-Yang-Mills, where the free energy scales as $\lambda N_{f} N_{c}$ instead of the naive $N_{f} N_{c}$ (see e.g. [25, 26, 27]). For the F1 string we see a very similar effect. This determination of the boundary entropy from the free energy contribution due to a probe brane can easily be generalized to higher dimensional systems. What is unclear to us at the moment is whether, as in $2 \mathrm{~d}$, in these higher dimensional examples an equivalent 
definition of the boundary entropy can also be given via the entanglement entropy. We hope to return to this issue in the future.

\subsection{Size and Shape (In)dependence}

Given our understanding of the meaning of the boundary entropy, we would expect that the contribution to the boundary entropy of a defect should be independent of the size of the subsystem enclosing the defect as long as the defect is in the center of the interval. For a defect that is off-center the folding trick can not be used to reduce the entanglement entropy calculation in the DCFT to the well known case of a BCFT, as we pointed out in section 2.2. It appears that in a DCFT the entanglement entropy of such an asymmetrically shaped region depends explicitly on the microscopic details and not just on the two universal numbers $c$ and $g$. In this subsection we want to reanalyze this issue in the context of the holographic calculation in the Janus framework.

We calculate the entanglement entropy of a spatial interval of length $l$ containing the defect on the field theory side, but potentially off-center. From the three-dimensional point of view, we must find the geodesic length of a spacelike segment $r(y)$ in the metric (3.2) with one endpoint at $y=-\infty, r=r_{0}$ and one endpoint at $y=\infty, r=r_{0}+\Delta r$. In line with what we said above, we expect the boundary entropy to be independent of the length $r_{0}$ but to depend on the asymmetry of the interval about the defect parameterized by $\Delta r$.

The geodesic action is independent of $r$ and leads to the conservation equation

$$
\frac{f(y) r^{\prime}}{\sqrt{f(y) r^{\prime 2}+1}}=\alpha
$$

where $\alpha$ is some constant that sets the asymmetry of the interval that is nontrivially related to $\Delta r$ (this can be seen by integrating $r^{\prime}$ from $y=-\infty$ to $y=\infty$ ).

The fact that the geodesic length depends only on $r^{\prime}$ tells us immediately that the boundary entropy is independent of $r_{0}$ as expected. In order to establish the dependence on $\alpha$, we must calculate the geodesic length in the Janus system for some nonzero $\alpha$ and subtract from it the geodesic length in the pure AdS system, being careful that in both calculations the boundary interval has the same length. It is very easy to see that in

this case the difference in entanglement entropies between Janus and pure AdS gets a contribution from the detailed shape of the warpfactor around the center of AdS (that is, around $y=0)$. 


\section{Conclusion}

In this paper we have calculated the boundary entropy in several strongly coupled $2 \mathrm{~d}$ defect conformal field theories which have a holographic dual. We confirmed that the definition of the boundary entropy in terms of the entanglement entropy gives identical answers to the definition in terms of a free energy at large temperature. Perhaps most interestingly, we found that this equivalence only holds in the case that one calculates the entanglement entropy for an interval that has the defect at the center, so that the DCFT can be mapped to a BCFT via the folding trick and the entanglement entropy is completely specified by two universal numbers, the boundary entropy and the central charge. In a DCFT, the entanglement entropy of an asymmetric interval captures detailed information about the microscopic details of the theory. In particular, from the knowledge of the entanglement entropy for arbitrarily shaped intervals one can reconstruct the length of all geodesics in the bulk and hence presumably the bulk metric.

Our methods employed in the bulk can readily be generalized to higher dimensions. In this case it is not clear if there is a similar universal definition of a boundary entropy as in $2 \mathrm{~d}$, though we may speculate that a coefficient of subleading divergent parts in the entanglement entropy will be a counterpart of the boundary entropy. However, it should still be interesting to calculate free energies and entanglement entropies associated with defects in strongly coupled theories in more than 2 dimensions.

\section{Acknowledgments}

AK would like to thank the Yukawa Institute for Theoretical Physics in Kyoto for their hospitality while this work was initiated. The work of AK and ET was supported in part by the U.S. Department of Energy under Grant No. DE-FG02-96ER40956. The work of TT is supported in part by JSPS Grant-in-Aid for Scientific Research No.18840027 and by JSPS Grant-in-Aid for Creative Scientific Research No. 19GS0219.

\section{A Janus Entropy at Weak Coupling from Free En- ergy}

\section{A.1 Two Point Function in the Presence of the Defect}

We consider the interface CFT defined by a $D$ dimensional free scalar field $\phi$ whose radius jumps from $R_{+}$to $R_{-}$at $y=0$. This is defined by the following action (in Euclidean 
space)

$$
S=\frac{R_{-}^{2}}{2} \int_{y<0} d^{D-1} x d y \partial_{\mu} \phi_{-} \partial_{\mu} \phi_{-}+\frac{R_{+}^{2}}{2} \int_{y>0} d^{D-1} x d y \partial_{\mu} \phi_{+} \partial_{\mu} \phi_{+}
$$

where $y$ is the direction which is perpendicular to the defect, $D$ is the total dimension and $\mu$ runs over all spacetime directions except $y$. We define

$$
c_{ \pm}=\frac{1}{R_{ \pm}^{2}}
$$

Imposing the on-shell condition, variation of the action with respect to $\delta \phi_{ \pm}$leads to

$$
\delta S=\left.\left[\frac{1}{c_{+}} \partial_{y} \phi_{+} \delta \phi_{+}-\frac{1}{c_{-}} \partial_{y} \phi_{-} \delta \phi_{-}\right]\right|_{y=0}
$$

Since we require the boundary condition

$$
\phi_{+}\left(x_{\mu}, y=0\right)=\phi_{-}\left(x_{\mu}, y=0\right) \text {, }
$$

we have $\delta \phi_{+}=\delta \phi_{-}$at $y=0$. Thus the principle of least action leads to

$$
c_{-} \partial_{y} \phi_{+}\left(x_{\mu}, y=0\right)=c_{+} \partial_{y} \phi_{-}\left(x_{\mu}, y=0\right)
$$

These two conditions are enough to determine the propagators

$$
\begin{aligned}
\left\langle\phi_{+}\left(x_{1}, y_{1}\right) \phi_{+}\left(x_{2}, y_{2}\right)\right\rangle & =\frac{c_{+}}{\left(\left(y_{1}-y_{2}\right)^{2}+\left(x_{1}-x_{2}\right)^{2}\right)^{\frac{D-2}{2}}}+\frac{c_{+} a_{+}}{\left(\left(y_{1}+y_{2}\right)^{2}+\left(x_{1}-x_{2}\right)^{2}\right)^{\frac{D-2}{2}}}, \\
\left\langle\phi_{-}\left(x_{1}, y_{1}\right) \phi_{+}\left(x_{2}, y_{2}\right)\right\rangle & =\frac{c_{+}\left(1+a_{+}\right)}{\left(\left(y_{1}-y_{2}\right)^{2}+\left(x_{1}-x_{2}\right)^{2}\right)^{\frac{D-2}{2}}}, \\
\left\langle\phi_{+}\left(x_{1}, y_{1}\right) \phi_{-}\left(x_{2}, y_{2}\right)\right\rangle & =\frac{c_{-}\left(1+a_{-}\right)}{\left(\left(y_{1}-y_{2}\right)^{2}+\left(x_{1}-x_{2}\right)^{2}\right)^{\frac{D-2}{2}}}, \\
\left\langle\phi_{-}\left(x_{1}, y_{1}\right) \phi_{-}\left(x_{2}, y_{2}\right)\right\rangle & =\frac{c_{-}}{\left(\left(y_{1}-y_{2}\right)^{2}+\left(x_{1}-x_{2}\right)^{2}\right)^{\frac{D-2}{2}}}+\frac{c_{-} a_{-}}{\left(\left(y_{1}+y_{2}\right)^{2}+\left(x_{1}-x_{2}\right)^{2}\right)^{\frac{D-2}{2}}},
\end{aligned}
$$

where we defined

$$
a_{+}=-a_{-}=\frac{c_{-}-c_{+}}{c_{-}+c_{+}}
$$

It is clear from the above formulation that we can equivalently treat the system such as two fields $\phi_{+}$and $\phi_{-}$that live in the same half space defined by $y \geq 0$. This is called the doubling (or folding) trick and it is rather common, especially in two dimensional CFTs. From this perspective, the constraints (A.4) and (A.5) are regarded as the NeumannDirichlet boundary condition at the open boundary $y=0$. 


\section{A.2 The Boundary Entropy in the Presence of the Defect}

Now we concentrate on the $D=2$ case. In order to compute the boundary entropy, we need to evaluate the partition function. We employ the normalized field $\varphi_{ \pm}=R_{ \pm} \phi_{ \pm}$such that the action looks like

$$
\begin{aligned}
S & =\int_{y>0} d t d y\left[\partial_{\mu} \varphi_{+} \partial_{\mu} \varphi_{+}\right]+\int_{y<0} d t d y\left[\partial_{\mu} \varphi_{-} \partial_{\mu} \varphi_{-}\right] \\
& =-\int_{y>0} d t d y\left[\varphi_{+} \partial_{\mu} \partial_{\mu} \varphi_{+}\right]-\int_{y<0} d t d y\left[\varphi_{-} \partial_{\mu} \partial_{\mu} \varphi_{-}\right]
\end{aligned}
$$

The boundary condition now becomes

$$
R_{-} \varphi_{+}=R_{+} \varphi_{-}, \quad R_{+} \partial_{y} \varphi_{+}=R_{-} \partial_{y} \varphi_{-}
$$

at the interface $y=0$. Imposing (A.9) and the following normalization,

$$
\int_{0}^{\infty} \varphi_{p}^{(i)+}(y) \overline{\varphi_{q}^{(j)+}(y)} \frac{d y}{2 \pi}+\int_{-\infty}^{0} \varphi_{p}^{(i)-}(y) \overline{\varphi_{q}^{(j)-}(y)} \frac{d y}{2 \pi}=\delta(p-q) \delta_{i j},
$$

we obtain the orthogonal basis with the momentum $p>0$ as follows 8

$$
\begin{aligned}
& \varphi_{p}^{(1)_{+}}(y)=\frac{\nu-1}{\sqrt{2\left(1+\nu^{2}\right)}} e^{i p y}+\frac{\nu+1}{\sqrt{2\left(1+\nu^{2}\right)}} e^{-i p y}, \\
& \varphi_{p}^{(1)_{-}}(y)=\frac{1-\nu}{\sqrt{2\left(1+\nu^{2}\right)}} e^{i p y}+\frac{\nu+1}{\sqrt{2\left(1+\nu^{2}\right)}} e^{-i p y},
\end{aligned}
$$

and

$$
\begin{aligned}
\varphi_{p}^{(2)_{+}}(y) & =\frac{\nu+1}{\sqrt{2\left(1+\nu^{2}\right)}} e^{i p y}+\frac{\nu-1}{\sqrt{2\left(1+\nu^{2}\right)}} e^{-i p y} \\
\varphi_{p}^{(2)-}(y) & =\frac{1+\nu}{\sqrt{2\left(1+\nu^{2}\right)}} e^{i p y}+\frac{1-\nu}{\sqrt{2\left(1+\nu^{2}\right)}} e^{-i p y} .
\end{aligned}
$$

Here, we defined $\nu=\frac{R_{+}}{R_{-}}$and the dependence on the time $x^{0}=t$ has been suppressed.

\footnotetext{
${ }^{8}$ It is useful to note the step function is expressed as follows

$$
\theta(y)=\frac{1}{2 \pi} \int \frac{d p}{i p+\epsilon} e^{i p y},
$$

which leads to

$$
\int_{0}^{\infty} \frac{d y}{2 \pi} e^{i p y}=\frac{1}{-i p+\epsilon}=\frac{1}{2} \delta(p)+i \frac{p}{p^{2}+\epsilon^{2}}
$$

Notice also

$$
\delta(p)=\frac{1}{-i p+\epsilon}+\frac{1}{i p+\epsilon} .
$$


When we expand the scalar field in terms of this basis

$$
\varphi=\sum_{p>0}\left(c_{p}^{(1)} \varphi_{p}^{(1)}+c_{p}^{(2)} \varphi_{p}^{(2)}\right)+c_{0} \varphi_{0}
$$

then the measure of the path-integral is given by

$$
[D \varphi]=\left(\prod_{p>0}\left[d c_{p}^{(1)}\right]\left[d c_{p}^{(2)}\right]\right) \cdot\left[d c_{0}\right] .
$$

On the other hand, in the case of the ordinary scalar field theory without any interface, the normalized basis is given by $e^{i p x}$ (just setting $\nu=0$ ). The measure for this basis is denoted as

$$
\left[D \varphi^{0}\right]=\left(\prod_{p>0}\left[d c_{p}^{0(1)}\right]\left[d c_{p}^{0(2)}\right]\right) \cdot\left[d c_{0}^{0}\right] .
$$

It is easy to see $\left[d c_{p}^{0(i)}\right]=\left[d c_{p}^{(i)}\right]$ from (A.14 and A.15). In this way we have found that the difference between the partition function with and without the defect comes from the $p=0$ contribution. Notice that the zero-mode $c_{0}^{(i)}$ spans the interval

$$
0 \leq c_{0}^{(i)} \leq \sqrt{2} \pi \sqrt{R_{+}^{2}+R_{-}^{2}}
$$

This is because the scalar $\varphi_{ \pm}$is compactified with the radius $R_{ \pm}$.

What we are interested in is the ratio

$$
g=\frac{Z_{\text {interface }}}{\sqrt{Z_{R_{+}} Z_{R_{-}}}}
$$

where $Z_{R_{+}}$is the partition function with an infinitely long $x$ direction radius with $R_{+}$. This clearly coincides with the ground state degeneracy $g$ discussed in this paper. In this ratio, the nonzero-modes, i.e. $c_{p}^{(i)}$, cancel out completely. The zero-mode contribution in the $x$ direction reads

$$
g=\frac{\sqrt{R_{+}^{2}+R_{-}^{2}}}{\sqrt{\left(\sqrt{2} R_{+}\right) \cdot\left(\sqrt{2} R_{-}\right)}}=\sqrt{\frac{R_{+}^{2}+R_{-}^{2}}{2 R_{+} R_{-}}} .
$$

\section{References}

[1] I. Affleck and A. W. W. Ludwig, Universal noninteger 'ground state degeneracy' in critical quantum systems, Phys. Rev. Lett. 67 (1991) 161-164. 
[2] P. Calabrese and J. L. Cardy, Entanglement entropy and quantum field theory, J. Stat. Mech. 0406 (2004) P002 hep-th/0405152.

[3] S. Yamaguchi, Holographic RG flow on the defect and $g$-theorem, JHEP 10 (2002) 002 hep-th/0207171].

[4] S. Ryu and T. Takayanagi, Holographic derivation of entanglement entropy from AdS/CFT, Phys. Rev. Lett. 96 (2006) 181602 hep-th/0603001.

[5] S. Ryu and T. Takayanagi, Aspects of holographic entanglement entropy, JHEP 08 (2006) 045 hep-th/0605073.

[6] C. Bachas, J. de Boer, R. Dijkgraaf and H. Ooguri, Permeable conformal walls and holography, JHEP 06 (2002) 027 hep-th/0111210.

[7] C. Holzhey, F. Larsen and F. Wilczek, Geometric and renormalized entropy in conformal field theory, Nucl. Phys. B424 (1994) 443-467 hep-th/9403108.

[8] H. Casini and M. Huerta, A finite entanglement entropy and the c-theorem, Phys. Lett. B600 (2004) 142-150 hep-th/0405111.

[9] T. Hirata and T. Takayanagi, AdS/CFT and strong subadditivity of entanglement entropy, JHEP 02 (2007) 042 hep-th/0608213.

[10] M. Headrick and T. Takayanagi, A holographic proof of the strong subadditivity of entanglement entropy, arXiv:0704.3719 [hep-th].

[11] L. Randall and R. Sundrum, An alternative to compactification, Phys. Rev. Lett. 83 (1999) 4690-4693 [hep-th/9906064.

[12] A. Karch and L. Randall, Locally localized gravity, JHEP 05 (2001) 008 hep-th/0011156.

[13] D. Bak, M. Gutperle and S. Hirano, A dilatonic deformation of AdS(5) and its field theory dual, JHEP 05 (2003) 072 [hep-th/0304129].

[14] D. Bak, M. Gutperle and S. Hirano, Three dimensional janus and time-dependent black holes, JHEP 02 (2007) 068 hep-th/0701108.

[15] A. Karch and E. Katz, Adding flavor to AdS/CFT, JHEP 06 (2002) 043 hep-th/0205236. 
[16] J. Babington, J. Erdmenger, N. J. Evans, Z. Guralnik and I. Kirsch, Chiral symmetry breaking and pions in non-supersymmetric gauge / gravity duals, Phys. Rev. D69 (2004) 066007 hep-th/0306018.

[17] S. Elitzur, E. Rabinovici and G. Sarkissian, On least action D-branes, Nucl. Phys. B541 (1999) 246-264 hep-th/9807161.

[18] C. Bachas and I. Brunner, Fusion of conformal interfaces, arXiv:0712.0076 [hep-th].

[19] D. Z. Freedman, C. Nunez, M. Schnabl and K. Skenderis, Fake supergravity and domain wall stability, Phys. Rev. D69 (2004) 104027 hep-th/0312055].

[20] A. B. Clark, D. Z. Freedman, A. Karch and M. Schnabl, The dual of janus ( $(i:)^{-} i^{-} \dot{z}$ (:z)) an interface CFT, Phys. Rev. D71 (2005) 066003 hep-th/0407073.

[21] A. Karch and L. Randall, Open and closed string interpretation of SUSY CFT's on branes with boundaries, JHEP 06 (2001) 063 hep-th/0105132.

[22] C. Bachas and M. Petropoulos, Anti-de-Sitter D-branes, JHEP 02 (2001) 025 hep-th/0012234.

[23] J. Raeymaekers and K. P. Yogendran, Supersymmetric D-branes in the D1-D5 background, JHEP 12 (2006) 022 hep-th/0607150.

[24] N. Couchoud, Anti-de Sitter branes with Neveu-Schwarz and Ramond-Ramond backgrounds, JHEP 03 (2003) 007 hep-th/0301195.

[25] D. Mateos, R. C. Myers and R. M. Thomson, Holographic phase transitions with fundamental matter, Phys. Rev. Lett. 97 (2006) 091601 hep-th/0605046.

[26] T. Albash, V. G. Filev, C. V. Johnson and A. Kundu, A topology-changing phase transition and the dynamics of flavour, hep-th/0605088.

[27] A. Karch and A. O'Bannon, Chiral transition of $N=4$ super Yang-Mills with flavor on a 3-sphere, Phys. Rev. D74 (2006) 085033 hep-th/0605120. 